\title{
A sensitive search for Muonium-Antimuonium-Oscillation
}

L. Willmann ${ }^{a}$, R. Abela ${ }^{c}$, Y. Bagaturia ${ }^{f}$, W. Bertl ${ }^{c}$, B. Braun ${ }^{a}$, R. Engfer ${ }^{b}$, B. Fischer v.Weikersthal ${ }^{a}$, V. W. Hughes ${ }^{g}$, K. Jungmann ${ }^{a}$, D. Kampmann ${ }^{d}$, V. Karpuchin ${ }^{e}$, I. Kisele , A. Klaas ${ }^{d}$, S. Korenchenko ${ }^{e}$, N. Kuchinsky ${ }^{e}$, A. Leuschner $^{b}$,

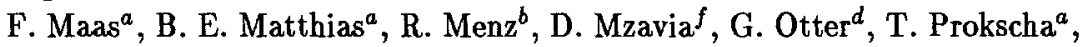
H. S. Pruys ${ }^{b}$, G. zu Putlitz ${ }^{a}$, W. Reichart ${ }^{b}$, I. Reinhard ${ }^{a}$, D. Renker ${ }^{c}$, T. Sakhelashvili ${ }^{f}$, P. Schmidt ${ }^{a}$, W. Schwarz ${ }^{a}$, R. Seeliger ${ }^{d}$, H. K. Walter ${ }^{c}$, L. Zhang ${ }^{a}$,

${ }^{a}$ Universität Heidelberg, D-69120 Heidelberg, Germany

${ }^{b}$ Universität Zürich, CH-8057 Zürich, Switzerland

${ }^{c}$ Paul Scherrer Institut, CH-5234 Villigen, Switzerland

${ }^{d}$ RWTH Aachen, D-52056 Aachen, Germany

${ }^{e}$ Joint Institute for Nuclear Research, RU-141980 Dubna, Russia

$f$ Tblisi State University, 380086 Tblisi, Georgia

${ }^{g}$ Yale University, New Haven, Connecticut 06520, USA

July 4,1994

\begin{abstract}
In the standard model lepton number violating processes are forbidden, but are allowed in many extensions to it. Muonium to antimuonium conversion has been explicitly allowed in the framework of left-right-symmetric models or in supersymmetric models. A new experiment at PSI has demonstrated its ability to improve the sensitivity to such a rare process significantly.
\end{abstract}

Recently the interest on lepton number violating processes has increased, because they are sensitive to new physics beyond the standard model. Of particular interest in this context is the muonium atom $\left(M \equiv \mu^{+} e^{-}\right)$, the bound state of a positive muon $\mu^{+}$and an electron $e^{-}$. The process of spontaneous conversion of muonium into its antiatom, antimuonium $\left(\bar{M} \equiv \mu^{-} e^{+}\right)$, violates additive muon and electron number conservation by two units each.

Herczeg and Mohapatra have derived a lower bound for the $M \longrightarrow \vec{M}$ effective conversion coupling in a minimal left-right symmetric model [1]. This process in which a doubly charged higgs boson $\Delta^{++}$is exchanged (see Fig. 1a) can be described as an effective four fermion interaction with a coupling constant $G_{M \bar{M}}$. From cosmological constraints on the lifetime of the muon neutrino $\nu_{\mu}$ (its mass should exceed $35 \mathrm{eV}$, which requires the particle to be unstable) and from the present upper limit on the energy density of the universe a lower limit of $G_{M \bar{M}}>7 \times 10^{-5} G_{F}$ [1] can be derived, assuming the present upper limit of $270 \mathrm{keV}$ for the muon neutrino mass. $G_{F}$ is the Fermi coupling constant of the weak interaction. If this limit can be lowered, the bound on $G_{M \bar{M}}$ increases.

$R$-parity violating supersymmetric theories are of particular interest among other models which have been discussed in the literature in connection with $M \longrightarrow \bar{M}$ conversion. In these models the conversion proceeds via the exchange of a $\tau$-sneutrino $\tilde{\nu}_{\tau}$, the 


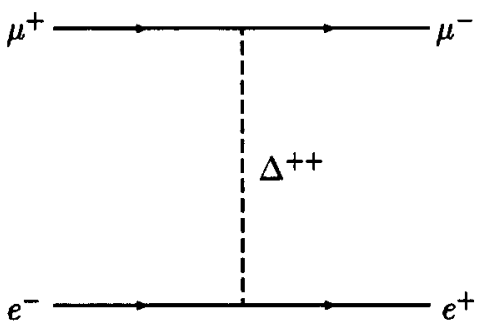

(a)

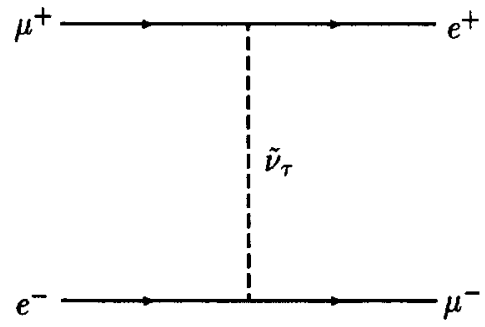

(b)

Figure 1: The $M \longrightarrow \bar{M}$ conversion mediated (a) by doubly charged Higgs boson $\Delta^{++}$in a left-right symmetric model and (b) by a $\tau$-sneutrino $\tilde{\nu}_{\tau}$ in R-parity violating supersymmetric models.

superpartner of the $\tau$-neutrino (see Fig. 1b). The effective coupling due to this process could be as large as $10^{-2}$ to $10^{-1} G_{F}[2]$.

Recent improvements on experimental possibilities to observe $M \longrightarrow \bar{M}$ conversion allow a stringent test of the theoretical approaches. A search, which is presently underway at PSI[3], utilizes the coincident detection of both constituents of antimuonium after its disintegration as a signature for the conversion [4]. A recent experiment at LAMPF [5] has demonstrated that this signature is superior to any other employed in searches to date. As a result $G_{M \bar{M}}<0.16 G_{F}$ (90\% C.L.) was obtained.

In the experiment at PSI muonium atoms are formed by electron capture after stopping a beam of positive muons of $21 \mathrm{MeV} / \mathrm{c}$ momentum in a $\mathrm{SiO}_{2}$ powder target. A fraction of the atoms leaves the surface of the powder target into the surrounding vacuum with thermal energies $(T=300(10))[6]$. Since the muonium yield in vacuum depends on the particular target and is known to fall back to half of its starting value on a time scale of 2 days the muonium production is monitored every four hours. The apparatus has been constructed to be as much as possible symmetric for the detection for muonium and antimuonium. A cylindrical magnetic spectrometer consisting of five concentric multi wire proportional chambers (MWPCs) and a segmented hodoscope covering $67 \%^{*} 4 \pi$ solid angle is used for the detection of the energetic $e^{-}\left(e^{+}\right)$from $\mu^{-}$ $\left(\mu^{+}\right)$decay. A new transport system was developed for the $e^{+}\left(e^{-}\right)$from the atomic shell of the respective atom which is left behind after decay of the antimuonium (muonium) atom. The particle is accelerated by a static electric field to $8 \mathrm{keV}$ kinetic energy and guided in magnetic field of $B=1 \mathrm{kG}$ parallel to the flux lines to conserve the transverse position information to a position sensitive microchannel plate (MCP) detector. It has a high transmission of $80 \%$ and allows a spatial resolution in the transverse direction better than $1 \mathrm{~mm}$. The position information is needed to distinguish between decays in the target and the nearby vacuum region. The MCP is surrounded by a pure CsI crystal detector consisting out of 12 segments which cover $75 \% * 4 \pi$ solid angle to detect the annihilation photons in antimuonium mode. Due to the symmetry in the detection of $M$ and $\bar{M}$ the apparatus can be calibrated with muonium decays in vacuum.

The feasibility of the experiment was tested in a first beam time in 1992 in the $\pi \mathrm{E} 3$ beam area of PSI. The apparatus was operated for approximatly one day searching for antimuonium decays in vacuum. We had $3.9(3) \cdot 10^{7}$ muonium atoms in the observation region. No antimuonium event could be identified. Taking into account correction factors representing differences in the relevant muonium respectively antimuonium de- 
tection efficiencies $(0.64)$, corrections for the finite observation time $(0.85)$, and the suppression of the $M \longrightarrow \bar{M}$ conversion in an external magnetic field $(0.35)$ we find at $90 \%$ confidence level the $M \longrightarrow \bar{M}$ conversion probability to be $P_{M \bar{M}} \leq 3.3 \cdot 10^{-7}$. The conversion probability is related to the coupling constant through $[3,5]$

$$
G_{M \bar{M}}=G_{F} \cdot \sqrt{\frac{P_{M \bar{M}}}{2.56 \cdot 10^{-5}}}
$$

In this first test we were able to establish a new experimental limit on the coupling constant of $G_{M \bar{M}}=0.11 G_{F}$ (90\%C.L.). This value is a slight improvement over the result obtained at LAMPF [5] previously and confirms it. Recently a value of $G_{M \bar{M}}<0.13 G_{F}$ (90\% C.L.) has been reported from an experiment at the JINR in Dubna which relies fully on the detection of a single electron from the $\mu^{-}$decay as the signature [7].

In a measurement period in summer 1993 we were able to search for $\approx 400$ hours for $M \longrightarrow \bar{M}$ conversion. Only part of the data has been analyzed so far, showing that we are now able to reproduce the results of previous experiments within 5 hours of data taking. This indicates that we can expect an increase in sensitivity to the conversion probability by two orders of magnitude corresponding to one order of magnitude in the sensitivity to the coupling constant.

In the near future further improvement in sensitivity of one order of magnitude is possible resulting from higher detection efficiency for the decay $e^{-}$(MWPC) and the atomic $e^{+}(\mathrm{MCP})$ which are achivable by standard technics. The experiment will also benefit from the availability of the new beam area $\pi E 5$ at PSI, where the muon flux is 5 to 10 times higher than the flux at the presently used area. These improvements should allow to put a stringent test on minimal left-right symmetric models, which predict a lower limit for the coupling constant $G_{M \bar{M}}$.

\section{Acknowledgements}

This work is supported in part by the BMFT, the Schweizer Nationalfond, and the Division of Scientific Affairs of NATO. One of us (L.W.) is grateful to the organizers of the conference for financial support.

\section{REFERENCES}

1. P. Herczeg and R. N. Mohapatra, Phys. Rev. Lett. 69, 2475 (1992), and references therein.

2. R. N. Mohapatra, Z. Phys. C56, 117 (1992); A. Halprin and A. Masiero, Phys. Rev. D48, 2987 (1993).

3. PSI Proposal R89-06.1 (1989); K. Jungmann and W. Bertl (spokespersons).

4. B. E. Matthias et al., Invited talk at Low Energy Muon Science Workshop Santa $\mathrm{Fe}, 1993$.

5. B. E. Matthias et al., Phys. Rev. Lett. 66, 2716 (1991).

6. K. A. Woodle et al., Z. Phys. D9, 59 (1988)

7. V.A. Gordeev et al. JETP Lett. 57, 270 (1993) 